\title{
Evaluating User-System Adaptability in the Use of Web-Based Information Systems through the Model of Access Efficiency
}

\author{
Bingcong Zeng* \\ Application and Business Services \\ Hewlett-Packard Enterprise, HK SAR Limited \\ City Plaza One, 1111 King's Road, Taikoo Shing, Hong Kong \\ anson.zeng@hpe.com \\ Benjamin P.-C. Yen \\ Faculty of Business and Economics \\ The University of Hong Kong \\ Pokfulam Road, Hong Kong \\ benyen@business.hku.hk
}

\begin{abstract}
Mutual adaptation between users and information systems as a significant research area is drawing more attention. This paper aims to construct an indicator to measure and evaluate the inter-adaptability between users and systems on the Web. We define inter-adaptability as the extent of match between users' preference and IS properties during the process of adaptation. It is vital to IS management since the quality of match between the two sides can dramatically influence the efficiency of IS use. In reality users and system are interactively adapting to each other in order to accommodate both sides' needs. However, most existing literature confines in the adaptation only on the system side. This study bridges the gap by taking into account the factors of both user-side and system-side adaptation, and for the first time connects the inlink properties with users' preference, offering a quick and practical measure to evaluate usersystem adaptability. By using this measure, practitioners can easily identify inefficient access problems in the exploitation of IS. To response to those problems identified, three strategies are proposed as a guideline for improvement. At the end, an empirical study on a blog system is elaborated, which shows the applicability of the model in practical use.
\end{abstract}

Keywords: Access Efficiency, Web-Based Information Systems, User-System Adaptability 


\section{Introduction}

Mutual adaptation between designers and users on the Web is an interesting and important research area. From designers' point of view, they would like to provide the best user experience of the system for every user, attempting to create enough use-cases so that each user's request can be satisfied without requiring the user to think consciously how to operate the system. However, the reality is rather uncertain. Designers are unable to know what users are truly looking for and thus they will tend to predict on users' behavior. As a result, the actual use of the system often differs from the designers' preconceptions, and information system (IS) becomes less helpful unless users and system both adapt till certain degree of user-system fit is reached (Poston and Speier, 2005). Accordingly, the performance of the adaptation process to some extent determines the efficiency of IS use.

Adaptation normally refers to a series of activities that users and system engage in either to make the system better fit its users or to change its users' behaviors for a better use of the system (Barki et al., 2007). For instance, when first using the system, users tend to acquire knowledge about the system structure. It may lead to a longer traveling path to access the resource they want. Gradually being more familiar with the system use, users will probably adjust their behaviors in choosing the traveling paths, having higher propensity to pick up the ones that are more convenient for them to reach the targets. Meanwhile, the system may dynamically manage the content as well as the structure to better accommodate with users' preference. As a whole, the adaptation process is a mutual interactive game between users and IS (Barki et al., 2007; Jansen, 2009). It proceeds iteratively until an equilibrium state is reached (Barki et al. 2007; Poston and Speier, 2005).

Instead of perceiving adaptation as a mutual interaction between users and system, traditional research emphasized system- side adjustments to accommodate users' needs. Such adaptation is achieved by providing customized services to enhance the effectiveness of navigation and improve user experience with information systems (Mohan et al., 1999). There are quite a number of studies on system-side adaptation. Tsandilas and Schraefel (2003) showed that adaptation of user interface can benefit users by alleviating the problem of information overload. Chen et al. (2005) contended that content adaptation to different technical environments (e.g. PC or wireless device) can effectively reduce users' navigation loss. In studying mobile web browsing behaviors, Adipat et al. (2011) found that involving users in the presentation adaptation is essential and allowing them to choose adaptation scheme can increase the ease of use. To better adapt the users in the Web navigation, Yen and Wan (2010) investigated various stochastic search based methods and proposed useful guidance for practitioners to follow.

With respect to adaptation techniques, there are three common approaches:

- Page splitting. The main idea of this method is to divide an original webpage into several sub-pages in order to fit users' specific requirements. The advantage is that it can significantly reduce information overload to the users; however, the disadvantage is that as the number of pages increases, the number of split pages will increase accordingly. Hence users have to spend more time on navigating the sub-pages (Zhang et al., 2014).

- Content summary. Users are allowed to browse the summary of web pages before deciding to visit any page with full details. This method spares the users from navigating unnecessary pages. However, it is not realistic to summarize all web pages and any inaccurate summary may lead to a 
wrong selection by the user (Olston and Chi, 2003; Smyth, 2009).

- Transcoding. This is an approach to tailoring web content in accordance with the capabilities of the client device and the network connection (Colajanni et al., 2005). It belongs to system-side adaptation to fit with user-side hardware needs (Nimmagadda et al., 2010). A typical example is to regenerate an image to lower resolution with the intention of reducing the size of the file to be downloaded.

The objective of system-side adaptation is to adjust IS, ranging from infrastructure to user interfaces, according to users' preferences, locations and behaviors. But meanwhile users also accustom themselves to the system, which however is often being neglected in the literature. As a matter of fact, the adaptation process is a mutual interaction between users and system (Barki et al., 2007; Jansen, 2009). Systemside adaptation only explains the adjustments of IS in response to the dynamic needs of users, but it fails to address users' adaptation to the system. Hence it may not fully reflect users' actual performance and their perception of the IS. To accurately describe the adaptation process, behaviors of both sides need to be considered (Barki et al., 2007).

The importance regarding user-system adaptation has been early identified in 1980s and 1990s. Gasser (1986) pointed out that the use of computer systems in organizations involves not only computing work but also adaptation work. Saga and Zmud (1994) suggested that adaptation activities should be taken into account in measuring the use of IS. Nevertheless, the research on adaptation process for analytics in the context of web-based systems is still at an initial stage, and it lacks indicators and evaluation methods to measure the adaptation performance. To bridge the gap, this study takes a close look at the adaptation process, and proposes the model of Access Efficiency $(A E)$ to reflect the intrinsic factors that have critical impacts upon the adaptation process. Briefly speaking, the $A E$ model is carefully designed to evaluate the user-system adaptability by assuming that there exists a degree of match between users and system in terms of access paths chosen. A good adaptation is able to direct users and system to a relatively high efficiency in information access while a poor one can lead to a rather lousy access performance. The model aims to depict the adaptation process and provide a measure to evaluate the adaptation performance.

The structure of this paper is organized as follows. First, we discuss the adaptation methods proposed in the existing literature and raise special concerns on two-sided adjustments between users and system during adaptation. We then analyze the adaptation process and derive the notions of user-system adaptability. Following this, we propose an analytical model of $A E$ with a simple numerical example. The score rating of $A E$ can effectively help practitioners to identify inefficient access problems in the IS easily. To improve $A E$, three strategies are proposed as a suggested guideline for practitioners in system design and administration. Finally, the model is empirically applied in analyzing the adaptation process in a blog system. The result shows the feasibility and the potential of the model in practical use.

\section{General Adaptation Process and User-System Adaptability}

In this section, we analyze the general adaptation process in the context of webbased IS by dividing the process into critical stages. Most of the information systems are implemented by using web technology nowadays. The so-called web-based information system essentially speaking is a website where information or knowledge is organized in an inter-linked network way for presentation. The adaptation of it normally 
happens in the early stage when the website is put into use. According to different objectives in adaptation, its general process can be conceptualized into two key phases: the initial technical adaptation phase and the content-structure adaptation phase.

In the initial technical adaptation phase, users browse to try out the functions when they first use the system. At this stage, users are more inclined to accommodate themselves to the technical aspect of the eenvironment, with their primary objectives of getting familiarized with the user interfaces and knowing how to locate and utilize various functions (Vandenbosch and Higgins, 1996). The time length of such technical adaptation will largely depend on users' IT skill (Holzinger et al., 2011; Lee et al., 1995). Users usually need to revisit the system several times in order to complete the initial technical adaptation. The process would be very painful for certain users with relatively insufficient IT knowledge. If serious, it might frustrate their intention to continuously use the IS in the future. But if high-quality of training about the system can be provided in advance, then the occurrence of this bad situation would be prevented to a great extent and the adaptation time could be shortened tremendously (Roca et al., 2006).

The content-structure adaptation phase, constituting the major part of the adaptation process, starts generally after or sometimes during the initial technical adaptation. In this phase, users browse the website with the aim to acquire information they desire. No doubt, one of the major functions of Webbased information system is to present and share information in a well-structured way. The "well-structured" here is referred to not only clear knowledge content shown in each page but also efficient links arranged among web pages. In order to obtain the information needed, users are required to go through some paths and reach certain web pages, which is very similar to performing searching tasks in the website. The performance of search tasks should be highly correlated with the organization of web content and the design of web structure, according to cognitive fit theory which believes that the task performance for individual users will be enhanced if there is a cognitive match between task and information presentation format (Vessey, 1991). In other words, if the web content and structure can be organized in high correspondence with the search tasks, then users will complete the tasks efficiently and capture the knowledge they want effectively (Beaudry and Pinsonneault, 2005). Goodhue and Thompson (1995) first identified the importance of the fit between information organization and task requirements, and conceptualized it as tasktechnology-fit (TTF). Barki et al. (2007) applied TTF in the study of IS-use related activities and claimed that there exists a degree of fit between users and system which has tremendous impact on the system use. Adopting the similar notion, User-system adaptability (U-S adaptability) in this paper specifies the concept under the context of adaptation. We define U-S adaptability as the extent of match between users' preference and the properties of webbased IS. The properties are referred to the attributes of the system that can be adjusted during the adaptation, such as the dynamic content of web pages and the link relationships among web pages. U-S adaptability can directly determine the effectiveness of users' browsing actions, thus influencing the efficiency of use of webbased IS.

In the content-structure adaptation phase, users and system mutually adapt to each other to pursue a higher degree of U-S adaptability. The effect involves the forces from two sides. On the system side, the content and web structure are no longer static. System designers or administrators would keep on reviewing and modifying the content and structure to enhance system adaptability to the users. For example, content summary is provided for users to preview so that they can decide whether to further read the detail content beforehand. 
Page splitting is also a good approach to improving adaptation on the system side. It can effectively reduce the chance of information overload to users. Moreover, many web-based information systems nowadays have already adopted dynamic web technology, such as PHP and JSP. So the system itself is able to adjust links among web pages to match up with the preference of its users. On the user side, through repetitive interaction with the system, users become increasingly familiar with the content and structure. They are able to decide more efficiently on the paths leading to their target pages. Consequently, the paths with higher efficiency are expected to gain more clickstream, which would eventually result in stable access patterns. At the end of the content-structure adaptation, users usually follow their preferred paths to complete tasks.

\section{Conceptual Development and Model Building}

The Access Efficiency $(A E)$ model is developed to reflect the inter-adaptability between users and system in terms of path selection to access web pages. In the process of mutual adaptation, while users gain more knowledge about the web content and structure, the system also adjust itself to accommodate dynamic users' preference. The access to webpage will become more efficient in normal cases when the adaptation proceeds. It is because users have higher propensity to choose shorter ways to complete tasks, hence probably creating more traffic for those shorter paths. $A E$ is designed to trace this trend and check whether users and system are in good match with each other for information access.

The model of $A E$ takes into account the factors for both system side and user side. On system side, the easiness of being accessed for web content and structure is modeled as so-called "accessibility" here. Accessibility of a webpage refers to how easily the page can be reached. If the webpage has more inlinks, it will be more convenient or easier for users to visit that page. Following this reasoning the number of inlinks could be regarded as an important measure for webpage accessibility. A model proposed by Yen (2007) suggested that the impact of each inlink on the accessibility be jointly determined by two factors: the attractiveness of the inlink and the level of its source page. Attractiveness is positively correlated with accessibility since the web page can be more easily accessed if the source link can draw more attention from users. The level of its source page is negatively correlated with accessibility because the deeper location of the web page is at, the longer time needed for users to take in order to reach. Based on that, the accessibility $A_{j}$ of destination page $j$ can be modeled as the summation of impacts from its all inlinks as below:

$$
A_{j}=\sum_{i=1}^{n} I_{i j}=\sum_{i=1}^{n}\left(\alpha_{i j} \times L\left(d_{i}\right)\right)
$$

where

- $I_{i j}$ is the impact of inlink $(i, j)$ on accessibility of destination page $j$, and $n$ is the total number of inlinks pointing to page j

- $d_{i}$ is the depth of page $i$, which is defined as the minimum number of links to follow from root page to page $i$. The level function $L\left(d_{i}\right)$ reflects the effect of depth on page accessibility and it is a decreasing function of $d_{i}$. For example, $L\left(d_{i}\right)=0.5^{\text {dit }}$

- $\alpha_{i j}$ measures the attractiveness of inlink (i, $j$ ) in source page $i$. If the inlink $(i, j)$ is highlighted with bright color or placed on the navigation bar, it will attract users to click this link. In our model, higher $\alpha_{i j}$

\footnotetext{
1 In Yen (2007), there are three types of decreasing function suggested. The article suggests using the exponentially decreasing function $0.5^{d i}$ to better reflect the impact brought by the page depth on Accessibility. We follow it in this study.
} 
implies the inlink receives more attention in the source page.

The conceptualization of accessibility above is based on the belief that the page that is being viewed currently imposes the most influence on visitors' behavior. The notion has been widely adopted in web analytics for its easiness in examining the path data without having to refer to the entire website (Jansen, 2009).

During adaptation, the web structure and user interfaces are periodically reviewed and revised by the designers, and dynamic links are established among web pages either manually or through automatic computing algorithms. As the result of these activities of system-side adaptation, the accessibilities of web pages are being changed in order to go well with users' preferences. Meanwhile, on user side, users are holding a subjective evaluation for each link. The more important they think the link is, the more likely they will select that link. For every webpage, there are multiple inlinks by which it can be accessed. Thereby for each inlink it is associated with a probability that reflects users' preference on it. For each visit, a specific inlink will be chosen. Accordingly, the actual impact of inlink on accessibility of page $j$ under user's judgment for a visit can be regarded as a random variable, which is denoted as $A l_{j}$ in the model. It is following a multinomial distribution with values $\left\{I_{1 j}, I_{2 j}, \ldots I_{n j}\right\}$ by their respective probability $\left\{p_{1 j}, p_{2 j}, \ldots p_{n j}\right\}$. The expected value of $A l_{j}$ is:

$$
E\left(A I_{j}\right)=\sum_{i=1}^{n} I_{i j} p_{i j}=\sum_{i=1}^{n}\left(\alpha_{i j} \times L\left(d_{i}\right)\right) \times p_{i j}
$$

where $p_{i j}$ is probability of inlink $(i, j)$ to chosen by users to access the destination page $j$. $\sum_{i=1}^{n} p_{i j}=1$ and $n$ is the total number of inlinks. The probability $p_{i j}$ can be measured by the usage rate of the inlink, which is equal to the clicks of inlink $(i, j)$ divided by the sum of clicks of total inlinks.
$E\left(A l_{j}\right)$ depicts the synergy of adaptation of both system-side and user-side. It represents the actual effective accessibility of page $j$. Mathematically $E\left(A l_{j}\right)$ should fall into the interval bounded based on the minimum and maximum value of the set $\left\{l_{1 j}\right.$, $\left.I_{2 j}, \ldots I_{n j}\right\}$. It varies in a spectrum when users update their preferences on the inlinks. Assume the inlinks have the same attractiveness (i.e. $\alpha_{i j}$ is the same). When $E\left(A l_{j}\right)$ is close to the lower bound of the spectrum, it indicates that users are more often using the ways of lower accessibility (e.g. longer paths) to access the destination page since higher probabilities are assigned to the inlinks which source pages are in deeper level. With $E\left(A l_{j}\right)$ increasing and moving towards the upper bound of the spectrum, it implies that users have better knowledge for accessing the target pages. They know the ways of higher accessibility (e.g. shorter paths) and use them more frequently. The position of $E\left(A l_{j}\right)$ in the spectrum, from the perspective of resource utilization, reflects the efficiency for users to access the page. It can be computed as follows:

$$
A E_{j}=\frac{E\left(A I_{j}\right)-\operatorname{Min}\left(S I_{j}\right)}{\operatorname{Max}\left(S I_{j}\right)-\operatorname{Min}\left(S I_{j}\right)}
$$

where $S I_{j}=\left\{I_{1 j}, I_{2 j}, \ldots I_{n j}\right\}$ (3)

Access Efficiency $A E_{j}$ measures how well users have done in choosing the paths to access the webpage $j$. It is positively correlated with U-S adaptability. When users and system become more mutual adapted, one critical indicator is that users are able to take more efficient way for information access. Since paths with higher efficiency are selected, $A E_{j}$ in general will be increased with the improvement of U-S adaptability. The value of $A E_{j}$ normally is between 0 and 1 as formula (3) implies. However, there are two extreme cases as exceptions:

- Exception 1: when the webpage $j$ has only 1 inlink, we define $A E_{j}$ to be 1 to indicate users have to select the 
only one link in source page to access the target page.

- Exception 2: when there is no visit to the webpage $j$, it will lead to the failure of forming probability distribution regarding inlink preference. To resolve it, we define the corresponding $A E_{j}$ to be 0 in this case. We can change the numerator in

$\operatorname{Max}\left(E\left(A I_{j}\right)-\operatorname{Min}\left(S I_{j}, 0\right)\right)$ reflecting its non-negativity. Unlike the situation that all users use the least efficient way (e.g. longest path) to visit the targeted page, it implies an even worse signal that some web resource is wasted or unable to be used.

\section{An Illustrated Example}

By using Access Efficiency, web designers can easily evaluate U-S adaptability during adaptation when changes are made in web structure. Suppose there is a website of only 7 pages, the structure of which is shown in Figure 1(a). For simplicity, we assume $L\left(d_{i}\right)$ is $0.5^{d i}$ and the importance of all inlinks $\alpha_{i j}$ is 1 . Given the clickstream traffic data shown on the arrows, we can obtain the accessibility and access efficiency of each webpage. It is found that Page 4 has multiple inlinks and its accessibility is high, however, $A E_{4}$ is below 0.5 which indicates that users are not using the most efficient way to access the page. Referred back to the traffic data, it manifests that users prefer taking path $\mathrm{H} \rightarrow 1 \rightarrow 4$ rather than $\mathrm{H} \rightarrow 4$. A similar case happens on Page 6 that most users choose the less efficient path $\mathrm{H} \rightarrow 2 \rightarrow 5 \rightarrow 6$. It is understandable that low values of $A E$ are shown at the initial phase of adaptation. When the adaptation process moves forward, users and system will mutually adapt to each other, as is shown in Figure 1(b). For Page 4, users gradually realize where the most efficient path is. More of them would like to take the path $\mathrm{H} \rightarrow 4$ instead of $\mathrm{H} \rightarrow 1 \rightarrow 4$. So $A E_{4}$ is increased from 0.4167 to 0.5833 . For Page 6 , the system shortens the access path from $\mathrm{H} \rightarrow 2 \rightarrow 5 \rightarrow 6$ to $\mathrm{H} \rightarrow 6$ in order to provide a more convenient way for users to take. The accessibility of Page 6 is doubled and the users notice that they can get access to Page 6 more easily. It shows that they accept the change and $A E_{6}$ surges from 0.25 to 0.75 correspondingly.

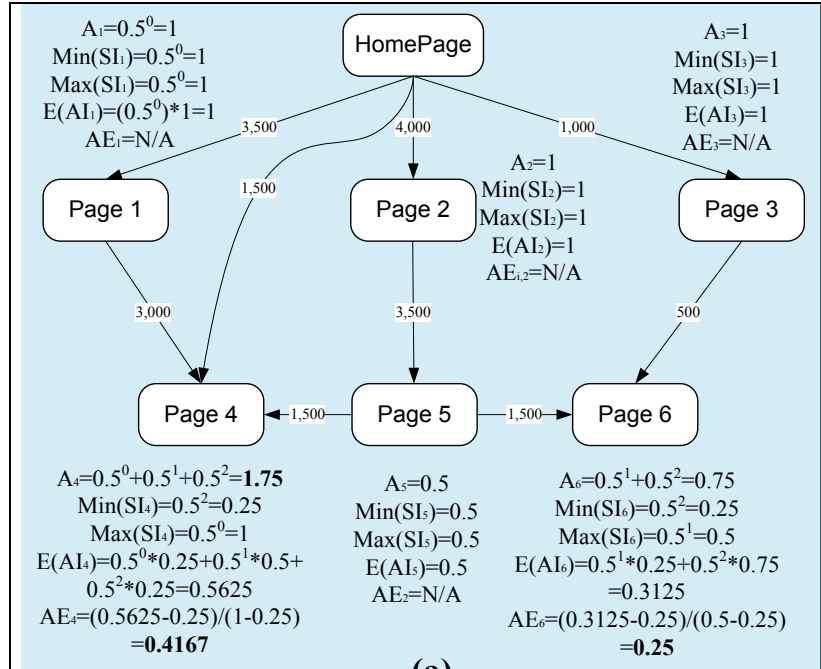

(a)

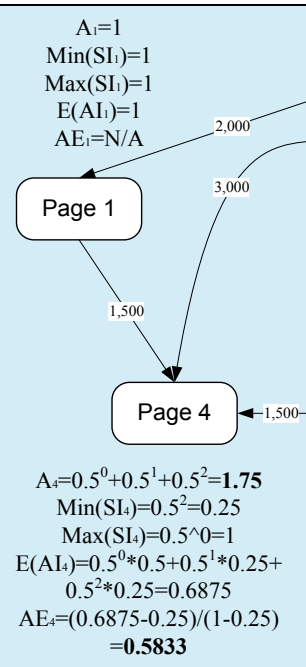

$=\mathbf{0 . 5 8 3 3}$

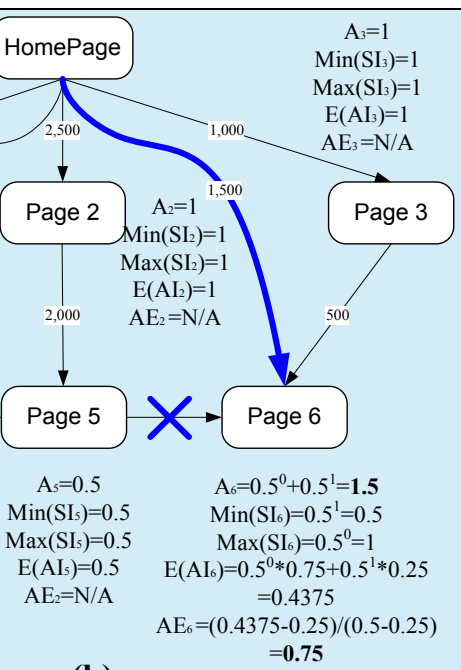

(b)

Figure 1 - Example of Using AE to Evaluate U-S Adaptability in Adaptation Process 


\section{Evaluation and Improvement Strategies}

$A E$ provides a quick and meaningful reflection of U-S adaptability in terms of the efficiency in using paths. The concept is based on the assumption that when U-S adaptability is high, the inlinks with stronger impact should receive more clickstream. In other words, accessibility impact $l_{i j}$, should positively correlate with probability $P_{i j}$ when users and system are well adapted with each other. The model would be more applicable under the context where browsing behaviors follow a customized sequential process. A typical case would be the e-Learning Management System (LMS), such as WebCT, Blackboard, and eCollege, in which students begin with a common start and then choose their preferred learning paths according to their interest, ability, current learning progress, etc. (Beldarrain, 2006). The score rating of $A E$ can help LMS administrators detect the problematic part of the e-learning system and determine where the inefficient access exists. The model has its limitation. It is not very good at analyzing the non-sequential navigation behaviors, like using search engine to locate information rather than clicking the links shown on web pages. The $A E$ ratio is still able to be computed under that situation, but the information revealed would probably be distorted from its original design.

In the model of $A E$, it is normal to see $I_{i j}$ and $P_{i j}$ are positively correlated when the $A E$ is greater than some level - activation level (e.g. 0.5). But if $A E$ is less than the activation level, it releases a signal that the correlation between $I_{i j}$ and $P_{i j}$ is low. Adaptation should be initiated again, otherwise the use of IS will become rather inefficient in such case. Once we confirm there is inefficient usage of inlinks to web pages by checking $A E$ to be less than the threshold value, plotting $I_{i j}$ and $P_{i j}$ in a graph can help to identify the problems and give useful hints on system-side adaptation. Essentially the plot of $I_{i j}$ and $P_{i j}$ (or $I-P$ plot) visually highlights the relationship between designers' anticipation and users' preference. Figure 2 below illustrates a simple example that could be seen in reality. The horizontal axis refers to the accessibility impact. The larger number the page gains in this dimension means it can be easier to access in the system. The vertical axis refers to the access probability. The higher value the page has implies the users are more likely to pay a visit to it. The point of $I-$ $P$ plot depicts the match of the visit expectation from the system view and the willingness of visit from users' view upon each page. Theoretically speaking, points are ideally to be found in regions I and III for higher U-S adaptability. The points in part 1, 2 and 3 bounded by the dash lines are improvable ones in terms of $A E$. To enhance the adaptation performance, three strategies are suggested respectively to the three improvable regions.

\section{- Strategy 1: Shorten the popular long paths}

The points in part 1 imply the inlinks that are quite inefficient but a great number of users would like to use them to visit the page. Inconvenience is the major issue here. To solve it, it is suggested to shorten those popular long paths, as it is done for page 6 in the example of Figure 1 - adding the inlink to create a short path to direct the traffic from the existing inlink associated with the long path.

\section{- Strategy 2: Make the inlinks more} attractive

The part 2 locates in the center of the graph, and the points within show no inclination towards either positive or negative correlation of $l_{i, j}$ and $p_{i, j}$. It means that the users using those inlinks may not be sensitive to web structure. It is suggested to increase $\alpha_{i j}$ by highlighting the inlinks in the source page or putting them in navigation bar in order to draw more attention from the users. The ultimate objective is to move more points to region I. 
- Strategy 3: Lengthen the short paths with few visits

The points in part 3 are associated with the inlinks with extremely high impact on destination page's accessibility but receiving very low clickstream. They are advised to be shorten in order to reduce $\operatorname{Max}\left(S l_{j}\right)$, and narrow the spectrum of $E\left(A l_{j}\right)$. The process of "lengthening" can be implemented by deleting the inlinks associated with short paths and adding the ones connected with long paths. For some extreme cases in which the visits are so small that their existence can be ignored, we suggest deleting those wasted inlinks.

The three strategies above are not limited to the situations with less than the activation level (e.g. 0.5). Another level, saturation level (e.g. 0.8), can be defined to denote the situation that no further improvement is necessary if their $A E$ is greater than the saturation level.

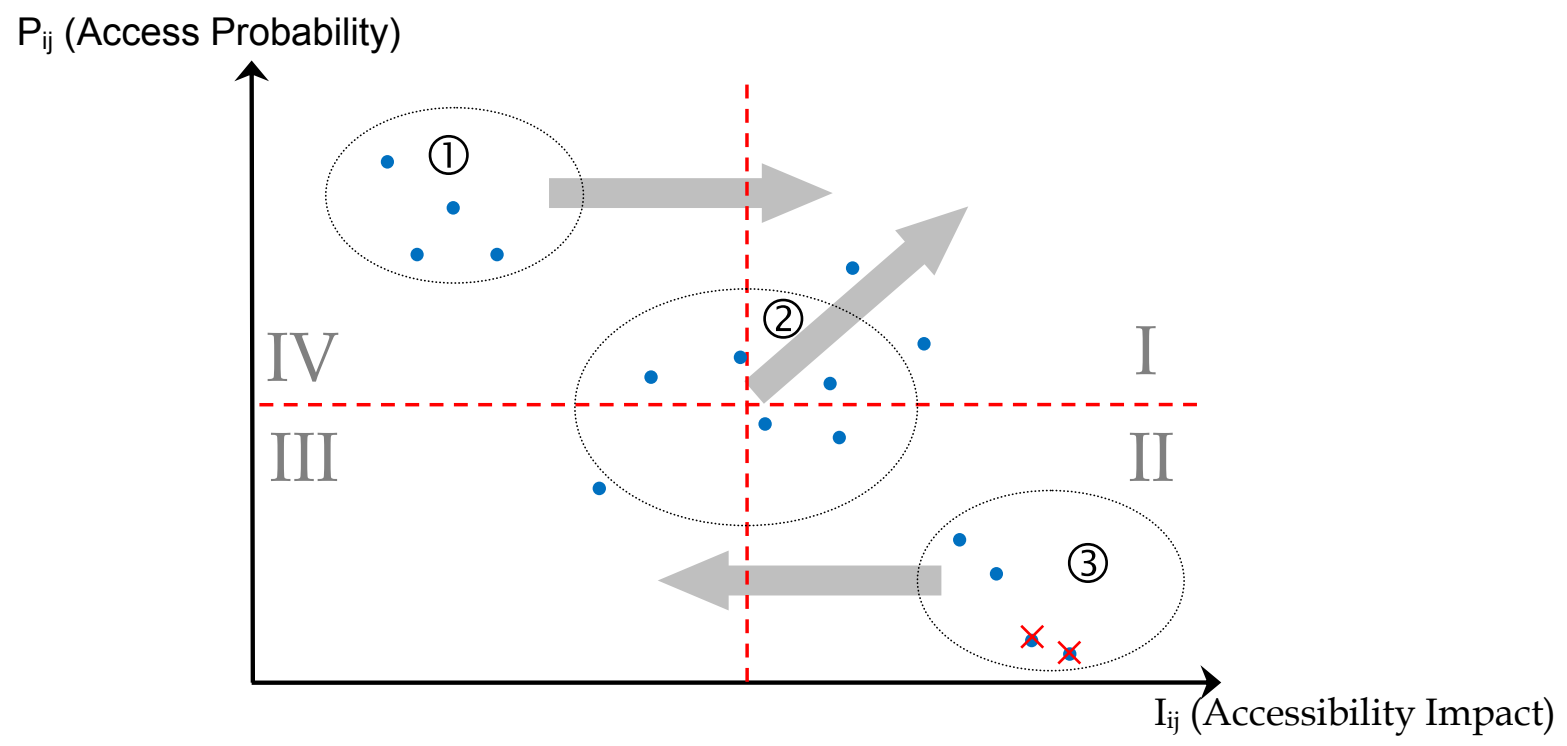

Figure 2 - I-P Plot Example and Three Strategies

\section{A Case Study: Applying Access Efficiency Analysis to Evaluate the User-System}

\section{Adaptability in a Blog Website}

An empirical study on a practical website has been conducted with the aim to use $A E$ to evaluate U-S adaptability. For convenience as well as easiness of data collection, a blog of WordPress (wordpress.org), one of the largest blog tools and publishing platforms, is chosen as the study subject. This blog adopts
WordPress's basic template which is implemented in PHP. It allows dynamic adaptation on system-side. For investigating the web structure, a Java application is developed that can crawl the website and intelligently record the site structure. There are 157 web pages of the blog crawled during the empirical study, 140 pages are included into the dataset, and 17 are excluded because they are advertisement or error message pages. The total number of links involved is 57,884 .

$A E$ analysis requires knowing the path traffic and the information of clickstream. To 
obtain the data needed, Google Web Analytics (http://www.google.com/analytics/), an online web analytics tool, is used in this study. The tool allows tracing the path traffic through page tagging. There are 140 pages in our dataset tagged with the JavaScript Google provided. When users access those pages, the JavaScript embedded will be triggered to send information about users' browsing actions and traveled pages to Google's remote servers. Google Web Analytics will process the data received and provide a comprehensive report on clickstream density for each webpage (See Figure 3). Although the click information on the report is of outlinks, visit numbers and jump-out rates are also available in Google Web Analytics which can help us to infer and compute the clickstream of the inlinks. Based on the report of Google Web Analytics, the clickstream distribution has been stable for the past week. It means the adaptation process is complete and the computed $A E$ can reflect the current situation of U-S adaptability.

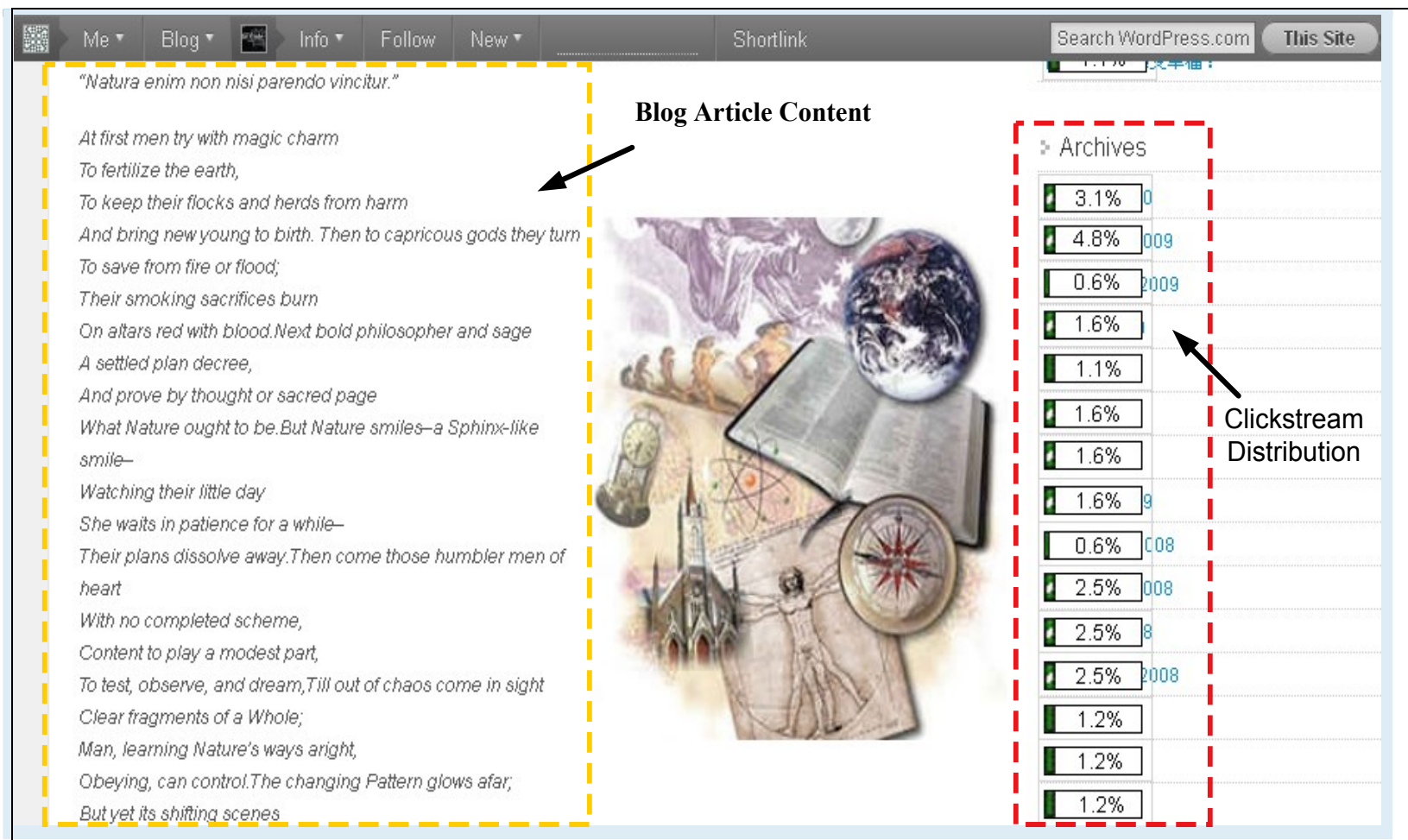

Figure 3 - A Snapshot of Click Density Report Generated by Google Web Analytics

Following the suggestion in Yen (2007), when computing $A E, 0.5^{d i}$ is used as the level function in the model. Moreover, to reflect the attractiveness of the link differentiated by different positions in the source page, if the link is shown in the navigation bar, $\alpha_{i j}$ is set to be 2 ; otherwise the value of 1 is assigned. A result summary of selected statistics of $A E$ analysis is presented in Table 1.
The overall U-S adaptability of this blog in terms of efficiency in using paths is good. The average $A E$ is 0.58 which manifests that the blog readers could access the desired information efficiently. $A_{j}$ and $A E_{j}$ are found highly correlated. It implies that the readers have a higher propensity to follow designers' anticipation in browsing the blog. Their browsing behaviors acutely depend upon the settings of the system. On the left side of Table 1 , it is interesting to find that 
all blog readers are using the link on the homepage to leave comments, resulting in the perfect efficiency for its access (See $A E_{j}$ of "/comments/feed/" in Table1). Moreover, for those on the most viewed list, the articles in the category of "Love Philosophy" and "Great Soul" could be easily accessed through the quick links on the homepage. Most readers prefer to use those quick links. Consequently it results in their very high values of $A E$. The page of "wp-login.php" enjoys extremely high accessibility (i.e. 89.1250 ) because nearly every page in this blog provides at least one link for users to login. The high $A E$ (i.e. 0.82) indicated that readers would like to log in the system when they just started to browse the blog. Since login often happened right after the visit of the homepage, the access to "wp-login.php" is quite efficient.

On the right side of Table 1 , there are 3 pages with $A E$ value of 0 , namely /2007/03/ index.php, /2007/03/index.php and /2007/03/ index.php. By tracing the log file, we note that these pages fail to receive any visits during the research. It is probably because they are all old articles and thereby readers had no incentive to pay any visit. Besides, the $A E$ of "/page/6/" is found extremely low. Figure 4 depicts its $I-P$ plot. The horizontal axis reflects how easy the page can be accessed and the vertical axis measures the traffic the page received. It reveals that two pages with powerful inlinks have very low traffic. If excluding the two outliers, $l_{i, j}$ and $P_{i, j}$ would show in a positive correlation, and all the points fall in a positively correlated region which is bounded in dash line on the left hand side. The page "/page/5/" has the similar problem as the one for "/page/6/". In fact, blog articles are by default displayed in chronological order, and these two pages with low $A E$ are the ones holding the oldest articles. In normal situations, readers do not use the quick links in the homepage to access "/page/5/" and "/page/6/". They prefer to read the articles page by page following time sequence. Thereby the access to the two old pages is inefficient as readers would probably not visit them through the quick ways. To increase their $A E$, we suggest using strategy 3 to lengthen the short paths with low visits. Referring to Figure 4, the two outliers are found to occupy less than $5 \%$ of the visits. Given that, we suggest deleting these pages. The deletion could help release the link space in the homepage or other highly accessible source pages, thus improving resource utilization efficiency of the whole system. Regarding the user-side adaptation, it is expected that users can access the resource they want in an easier way. Reflected in I-P plot, the positive correlation between $l_{i, j}$ and $P_{i, j}$ should increase. Because of the improvement of match between system's expectation and user's willingness, the adaptation time can be shortened tremendously.

\section{Table 1 - Result Summary of Selected Statistics of AE Analysis}

\begin{tabular}{|l|l|l|l|l|l|}
\hline Total Number of Web Pages: 140 & AVG $A E=0.58$ & \multicolumn{2}{c|}{$\operatorname{Corr}\left(\boldsymbol{A}_{j}, \boldsymbol{A} E_{j}\right)=0.7103$} \\
\hline \multicolumn{2}{|c|}{ Top5 Web Pages with Highest $A E_{j}$} & \multicolumn{2}{c|}{ Top5 Web Pages with Lowest $A E_{j}$} \\
\hline URL & $\boldsymbol{A}_{j}$ & $\boldsymbol{A} E_{j}$ & URL & $\boldsymbol{A}_{j}$ & $\boldsymbol{A E}_{\boldsymbol{j}}$ \\
\hline /comments/feed/ & 26.6875 & 1 & $/ 2007 / 03 /$ index.php & 3.3125 & 0 \\
\hline /category/LovePhilopophy/index.php & 23.5000 & 0.86 & $/ 2007 / 04 /$ index.php & 3.3125 & 0 \\
\hline /category/GreatSoul/index.php & 23.5000 & 0.84 & $/ 2007 / 05 /$ index.php & 3.3125 & 0 \\
\hline /wp-login.php & 89.1250 & 0.82 & /page/6/ & 4.3100 & 0.16 \\
\hline /2009/09/22/a-weakness/ & 19.5625 & 0.81 & /page/5/ & 4.5500 & 0.17 \\
\hline
\end{tabular}




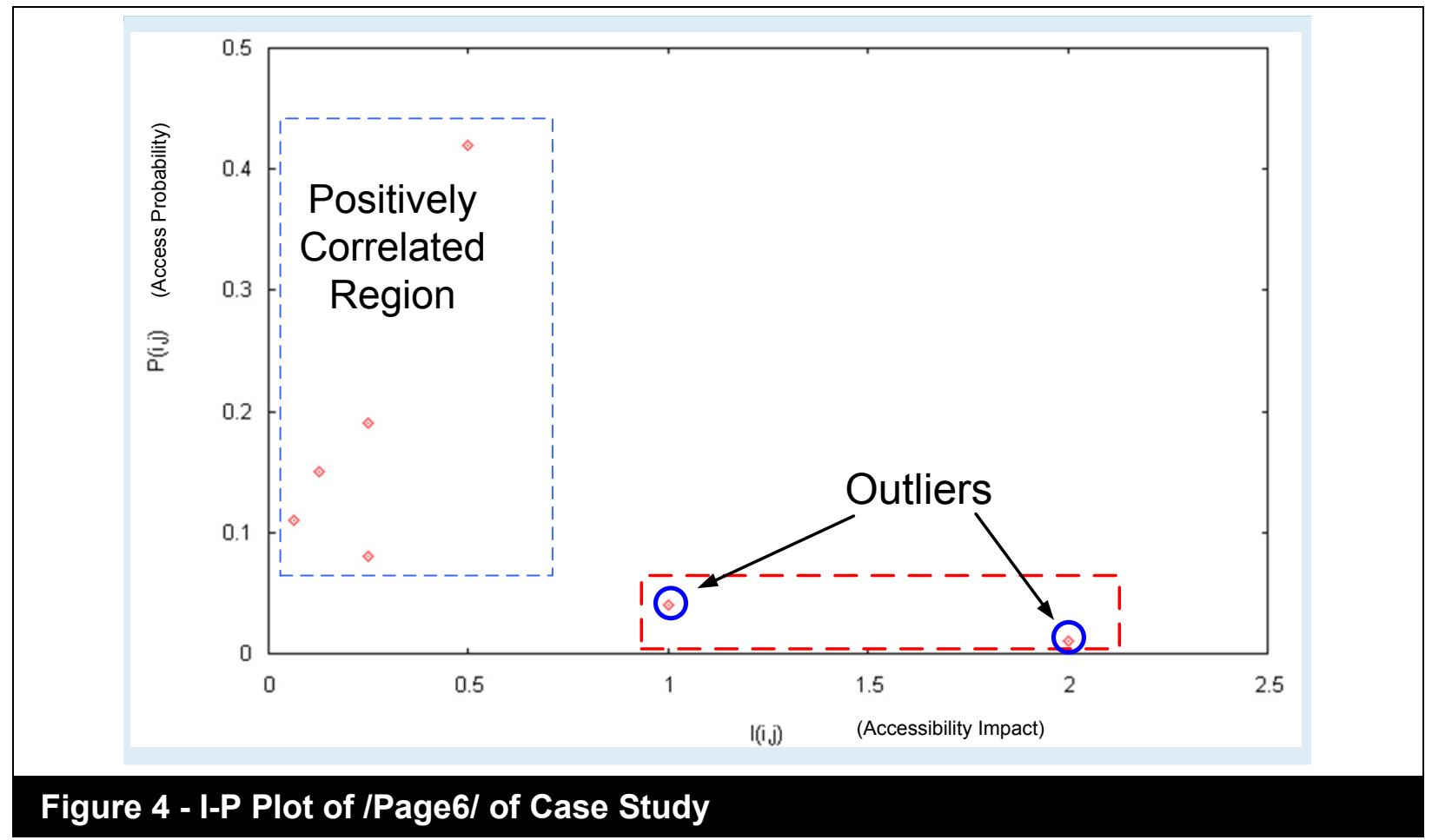

\section{Conclusion and Future Study}

Following the IS adoption phase, IS adaptation is another crucial stage that tremendously affects the effectiveness of IS use (Vessey, 1991; Goodhue and Thompson, 1995; McGill and Klobas, 2009). It covers a series of activities that users and system are both engaged in either to make the system better fit its users or to change the users' behaviors for a better use of the system (Barki et al., 2007). Therefore, the adaptation process in general is a mutual interactive game between users and IS (Barki et al., 2007; Jansen, 2009). In this study, we develop the concept of usersystem adaptability, which is defined as the extent of match between IS properties and users' preference during adaptation. We contend that the quality of adaptation is subject to the level of degree of U-S adaptability, and the improved U-S adaptability is manifested in the increasing efficiency of information access. Following this reasoning, we further propose the model of Access Efficiency to evaluate U-S adaptability under the use of web-based information systems. The feasibility of the model in practical use is tested and demonstrated through an empirical case analysis on the adaptation process of a blog system.

Our research has several important contributions for theory and practice. First, the concept of U-S adaptability we propose in this paper extends the concept of adaptation and customizes this insightful concept in the context of IS adaptation. It will enrich and improve the understanding of the adaptation process between users and system, especially in the content-structure adaptation phase. Second, most existing literature only limits in the study of adaptation on the system side, overlooking the effect made by users' adaptation. Our research bridges the gap by comprehensively considering the factors of both user-side and system-side adaptation in the model. Different from traditional traffic analysis, it's for the first time connects the inlink properties with users' preference, offering a quick and meaningful snapshot of U-S adaptability from the resource utilization perspective. This may arouse rethinking the 
view of modeling in the field of IS adaptation. Third, although the model of $A E$ has its limitation in analyzing non-sequential navigation behaviors in the adaptation process, it is still widely applicable in lots of scenarios such as e-learning environment. Moreover, the model not only supports agile trace of path performance but also fits in large-scale and dynamic website analysis. By adopting $A E$ model and analysis, practitioners can easily identify the problematic web pages which are being inefficiently accessed. Furthermore, we suggest three strategies as a guideline for improving $A E$ in the paper. All of them are practical and easy to implement. They may benefit the practitioners in system design and daily administration.

In this study, one empirical case has been used to demonstrate the practical use of $A E$ model. We understand a single case is not enough to verify the robustness of the model. Hence, in future study a comprehensive experiment will be conducted to address this issue. Furthermore, a smooth and quick adaptation is greatly expected for a better utilization of information systems. The time length undoubtedly is one of the most critical factors in adaptation process. For future study, the time factor could be taken into account in the model of $A E$. The current study purely focuses on how to measure Access Efficiency and modify web content and structure to improve adaptation quality. However, regarding how to speed up the overall adaptation process, it still remains unclear and thereby this would be a very significant research direction to explore in the future.

\section{Acknowledgements}

We would like to thank the journal's editors and anonymous reviewers since the valuable suggestions they provided have made substantial improvements to this paper. The research was supported in part by Hong Kong RGC under grant GRF 719409E and The University of Hong Kong under grant Seed Funding for Basic Research 11-12.

\section{References}

Adipat, B., Zhang, D., and Zhou, L. (2011). "The Effects of Tree-View Based Presentation Adaptation on Model Web Browsing," MIS Quarterly 35(1), pp. 99-121.

Barki, H., Titah, R., and Boffo, C. (2007). "Information System Use-Related Activity: An Expanded Behavioral Conceptualization of Individual-Level Information System Use," Information Systems Research 18(2), pp. 173-192.

Beaudry, A., and Pinsonneault, A. (2005). "Understanding user responses to information technology: a coping model of user adaption," MIS Quarterly, 29(3), pp. 493-524.

Beldarrain, Y. (2006). "Distance Education Trends: Integrating New Technologies to Foster Student Interaction and Collaboration," Distance Education 27(2), pp 139-153.

Chen, Y., Xie, X., Ma, W.-Y., and Zhang, H.J. (2005). "Adapting Web Pages for Small-Screen Devices," IEEE Internet Computing 9(1), pp 50-56.

Colajanni, M., Lancellotti, R., and Yu, P.S. (2005). "Distributed Architectures for Web Content Adaptation and Delivery," in: Web Content Delivery, X. Tang, J. Xu and S.T. Chanson (eds.). New York: Springer, pp. 285-304.

Gasser, L. (1986). "The Integration of Computing and Routine Work," ACM Trans. on Office Inform. Systems 4(3), pp. 205-225.

Goodhue, D.L., and Thompson, R.L.(1995). "Task-Technology Fit and Individual Performance," MIS Quarterly 19(2), pp. 213-236. 
Holzinger, A., Searle, G., and Wernbacher, M. (2011). "The Effect of Previous Exposure to Technology on Acceptance and Its Importance in Usability and Accessibility Engineering," Universal Access in the Information Society 10(3), pp 245-260.

Jansen, B.J. (2009). "Understanding UserWeb Interactions Via Web Analytics," San Rafael, Calif.: Morgan \& Claypool Publishers.

Lee, S.M., Kim, Y.R., and Lee, J. (1995). "An Empirical Study of the Relationships among End-User Information Systems Acceptance, Training, and Effectiveness," Journal of Management Information Systems 12(2), pp. 189-203.

McGill, T.J., and Klobas, J.E. (2009). "A Task-Technology Fit View of Learning Management System Impact " Computers \& Education 52(2), pp. 496-508.

Mohan, R., Smith, J.R., and Li, C.-S. (1999). "Adapting Multimedia Internet Content for Universal Access," IEEE Transactions on Multimedia 1(1), pp. 104-114.

Nimmagadda, Y., Kumar, K. and Lu Y. (2010). "Adaptation of Multimedia Presentations for Different Display Sizes in the Presence of Preferences and Temporal Constraints," IEEE Transactions on Multimedia 12(7), pp. 650-664.

Olston, C., and Chi, E.H. (2003). "Scenttrails: Integrating Browsing and Searching on the Web," ACM Transactions on Computer-Human Interaction 10(3), pp. 188-197.

Poston, R.S., and Speier, C. (2005). "Effective Use of Knowledge Management Systems: A Process Model of Content Ratings and Credibility Indicators," MIS Quarterly 29(2), pp. 221-245.
Roca, J.C., Chiu, C.-M., and Martínez, F.J. (2006). "Understanding E-Learning Continuance Intention: An Extension of the Technology Acceptance Model," International Journal of Human-Computer Studies 64(8), pp 683-696.

Saga, V.L., and Zmud, R.W. (1994). "The Nature and Determinants of It Acceptance, Routinization, and Infusion," in: Diffusion, Transfer, and Implementation of Information Technology., L. Levine (ed.). Amsterdam, The Netherlands: Elsevier, pp. 67-86.

Smyth, B. (2009). "Case Studies in Adaptive Information Access: Navigation, Search, and Recommendation," in: Intelligent User Interfaces: Adaptation and Personalization Systems and Technologies C. Mourlas and P. Germanakos (eds.). Hershey, PA: Information Science Reference, pp. 35-59.

Tsandilas, T., and Schraefel, M.C. (2003). "Adaptive Presentation Supporting Focus and Context," in: Proceedings of the Workshop on Adaptive Hypermedia and Adaptive Web-Based Systems. Nottingham, UK, pp. 193205.

Vandenbosch, B., and Higgins, C.H. (1996). "Information Acquisition and Mental Models: An Investigation into the Relationship between Behavior and Learning," Information Systems Research 7(2), pp. 198-214.

Vessey, I. (1991). "Cognitive Fit: A TheoryBased Analysis of the Graphs Versus Tables Literatures," Decision Sciences 22(2), pp. 219-240.

Yen, B.P.-C. (2007). "The Design and Evaluation of Accessibility on Web Navigation," Decision Support Systems 42(4), pp. 2219-2235.

Yen, B.P.-C., and Wan, Y. (2010). "Design and Evaluation of Improvement 
Method on the Web Information Navigation - a Stochastic Search Approach," Decision Support Systems 49(1), pp. 14-23.

Zheng, Y., Burke, R. and Mobasher, B. (2014). "Splitting approaches for context-aware recommendation: an empirical study," in: Proceedings of the 29th Annual ACM Symposium on Applied Computing, Gyeongju, Republic of Korea, pp. 274-279.

\section{About the Authors}

Bingcong Zeng is a technical constant in Hewlett-Packard Enterprise. $\mathrm{He}$ is responsible for investigating cloud computing in HP labs. He received his Ph.D. degree in Management Information Systems from The University of Hong Kong. His research mainly focuses on healthcare information systems, cloud computing, and IT auditing.

Benjamin P.-C. Yen is an Associate Professor in the School of Business at The University of Hong Kong. He received his Ph.D. degree in Industrial Engineering and Operations Research from Columbia University. His research interests include electronic catalogs in electronic commerce, IT based supply chain management, and Web information retrieval. He has papers published in major information system and operations research journals including IEEE Transactions on Systems, Man, and Cybernetic, Decision Support Systems, Journal of Organizational Computing and Electronic Commerce, Journal of Information Technology, Electronic Commerce Research Journal, Information Processing Letters, Annals of Operations Research, and European Journal of Operations Research, etc. 